\title{
Viability and Equilibrium in Securities Markets with Frictions*
}

\author{
Elyès JOUINI $^{\dagger} \quad$ Hédi KALLAL ${ }^{\ddagger}$ \\ June 1995, this version March 1999, \\ to appear in Mathematical Finance
}

\begin{abstract}
In this paper we study some foundational issues in the theory of asset pricing with market frictions. We model market frictions by letting the set of marketed contingent claims (the opportunity set) be a convex set, and the pricing rule at which these claims are available be convex. This is the reduced form of multiperiod securities price models incorporating a large class of market frictions. It is said to be viable as a model of economic equilibrium if there exist price-taking maximizing agents who are happy with their initial endowment, given the opportunity set, and hence for whom suplly equals demand. This is equivalent to the existence of a positive linear pricing rule on the entire space of contingent claims an underlying frictionless linear pricing rule - that lies below the convex pricing rule on the set of marketed claims. This is also equivalent to the absence of asymptotic free lunches - a generalization of opportunities of arbitrage. When a market for a non marketed contingent claim opens, a bid-ask price pair for this claim is said to be consistent if it is a bid-ask
\end{abstract}

${ }^{*}$ We have strongly benefitted from conversations with Kerry Back, Jean-Marc Bonnisseau, George Constantinides, Darell Duffie, Philip Dybvig, Ivar Ekeland, Lars Hansen, Chi-Fu Huang, Fulvio Ortu, Manuel Santos, David Schmeidler, Mike Woodford, and especially José Scheinkman. We are also grateful to seminar participants at the University of Chicago for helpful comments. The financial supports of NSF grant \# SES 9022643, the Santa Fe Institute, and of the Sloan Foundation are gratefully acknowledged. This research was developed while Kallal was affiliated with the University of Chicago and New York University. Salomon is not responsible for any statement or conclusions herein, and no opinions, theories, or techniques presented herein in any way represent the position of Salomon Brothers Inc.

${ }^{\dagger}$ CREST-ENSAE, 15 Boulevard Gabriel Peri, 92245 Malakoff Cedex, FRANCE.

$\ddagger$ Salomon Brothers, 7 World Trade Center, New York, 10048, NY, USA. 
price pair in at least a viable economy with this extended opportunity set. If the set of marketed contingent claims is a convex cone and the pricing rule is convex and sublinear, we show that the set of consistent prices of a claim is a closed interval and is equal (up to its boundary) to the set of its prices for all the underlying frictionless pricing rules. We also show that there exists a unique extended consistent sublinear pricing rule - the supremum of the underlying frictionless linear pricing rules - for which the original equilibrium does not collapse, when a new market opens, regardless of preferences and endowments. If the opportunity set is the reduced form of a multiperiod securities market model, we study the closedness of the interval of prices of a contingent claim for the underlying frictionless pricing rules.

KEYWORDS: Viability, equilibrium, absence of arbitrage, free lunch, market frictions, convex and sublinear pricing rule, consistent bid-ask prices, arbitrage bounds, equilibrium bounds.

\section{Introduction and synopsis}

In the foundational theory of asset pricing, developed in the Arrow-Debreu model (Theory of value [1959, chap. 7]), Black and Sholes (1973) and Cox and Ross (1976 a and b), and subsequently formalized in a general framework by Harrison and Kreps (1979), Harrison and Pliska (1979), Kreps (1981), and Duffie and Huang (1986), securities markets are assumed to be frictionless. The main result is that a securities price model is viable as a model of economic equilibrium, or equivalently is arbitrage-free, if and only if there exists a strictly positive linear pricing rule that prices the contingent claims in the opportunity set, modeled as a linear space.

In this paper, we develop some foundational issues related to the theory of asset pricing, in securities markets with frictions. We model market frictions by letting the set of marketed contingent claims $M$ (the opportunity set of available investments) be a convex set containing 0 - instead of a linear space as in the frictionless case - and by letting the pricing rule $\pi$ at which these contingent claims are available be a convex function satisfying $\pi(0)=0$ - instead of a linear function as in the frictionless case. The fact that the set of marketed contingent claims is convex means that if two contingent claims $x$ and $y$ are marketed, their average $\frac{1}{2}(x+y)$ is also marketed. The fact that the pricing rule is convex means that its price is lower than or equal to their average price. The convexity of $\pi$ and the fact that $\pi(0)=0$ imply that the price at which a contingent claim can be bought is larger than or equal to the price at which it can be sold. ${ }^{1}$ We consider this class of pricing rules, that includes the linear pricing rules, in order to take a large

\footnotetext{
${ }^{1}$ Indeed, $0=\pi(0)=\pi\left(\frac{x-x}{2}\right) \leq \frac{1}{2} \pi(x)+\frac{1}{2} \pi(-x)$ implies that $\pi(x) \geq-\pi(-x)$.
} 
class of market frictions into account. Indeed, this model is the reduced form of a multiperiod securities markets model where agents can trade a finite number of securities. These securities, which give the right to an amount of consumption contingent on the state of the world at the final date, are used to transfer consumption from the initial date to the final date. The set of marketed claims is then the set of contingent claims that can be obtained (or dominated) through securities trading while satisfying the constraints imposed by potential market imperfections (such as, for instance, short sales or borrowing constraints), as well as a budget constraint taking potential costs (such as, for instance, transaction costs, shortselling costs, borrowing costs or taxes) into account. The pricing rule is then defined for any given marketed contingent claim as the minimum cost of obtaining (or dominating) it using such a trading strategy. If there are no market frictions at all, the set of marketed claims is a linear subspace of the space of contingent claims, and the pricing rule is linear (see Harrison and Kreps [1979]). For a large class of market frictions - including bid-ask spreads, short sales constraints, shortselling costs, borrowing constraints, borrowing costs and taxes - it can be shown that the set of marketed claims is a convex cone and that the pricing rule is sublinear (see Jouini and Kallal [1995 a and b]). On the other hand, if transaction costs increase less than proportionally with transaction size, the set of marketed claims is a convex cone but the pricing rule is only convex. Also, in an economy where agents have risk limits on their portfolios the set of marketed claims and the pricing rule are only convex.

A price system, i.e. the combination of a convex set of marketed claims and of a convex pricing rule, is said to be viable as a model of economic equilibrium (a concept introduced by Harrison and Kreps [1979]) if there exist maximizing agents with a convex, continuous, and stricly increasing preorder of preferences who are happy with their endowment, i.e. for whom supply is equal to demand. We show that a price system is viable if and only if there exists a positive linear pricing rule on the entire space of contingent claims that lies below the convex pricing rule on the convex set of marketed claims (we call such a linear pricing rule an underlying frictionless linear pricing rule). This is in fact equivalent to the absence of asymptotic free lunches - a generalization of opportunities of arbitrage - in this type of economy. ${ }^{2}$

When a market for a non marketed contingent claim opens, we say that a bidask price pair is consistent if it is a bid-ask price pair in at least a viable economy with this extended opportunity set, and with identical or tighter bid-ask spreads

\footnotetext{
${ }^{2}$ In multiperiod securities price models, the underlying linear pricing rules can be shown to be equal to the martingale, supermartingale, or martingale measures of appropriately normalized price processes (or other related processes), depending of the nature of the market frictions (see Jouini and Kallal [1995 a and b]).
} 
on the set of originally marketed claims. If the set of marketed claims is a convex cone and the pricing rule is sublinear, we show that the set of consistent prices of a contingent claim is a closed interval and is equal (up to its boundary) to the set of its prices for all the underlying frictionless pricing rules. However, as a market for a non marketed contingent claim opens (and as bid-ask spreads on the originally marketed contingent claims are potentially made tighter), new investment opportunities may appear, and agents may reshuffle their portfolio causing the existing equilibrium to collapse. Nonetheless, we show that there exists a unique extended consistent sublinear pricing rule - namely the supremum of the underlying frictionless linear pricing rules - for which the original equilibrium does not collapse when a new market opens, no matter what preferences and endowments are. This means that without any further restrictions on preferences and endowments, and based on arbitrage and equilibrium arguments alone, we cannot infer tighter bounds on contingent claim prices than the arbitrage bounds also given by the underlying frictionless pricing rules. Indeed, any tighter bid-ask price interval could lead to a collapse of the equilibrium in place, in at least an economy.

In the case where the opportunity set is the reduced form of a multiperiod securities market model, we study the closedness of the interval of prices of a contingent claim for the underlying frictionless pricing rules. In particular, we show that if markets are incomplete but otherwise frictionless this set is closed if and only if it is reduced to a single element (a result of Jacka [1992] and Ansel and Stricker [1994]).

Related work includes Leland (1985), Dybvig and Ross (1986), Prisman (1986), Ross (1987), Back and Pliska (1990), Bensaid et al. (1991), Hindy (1991), He and Pearson (1991), Cvitanic and Karatzas (1993), and Constantinides (1993). An important part of the mathematical tools used in this paper are presented in Schachermayer (1993) and in Delbaen and Schachermayer (1994a).

The remainder of the paper is organized as follows. Section 2 characterizes viability and the absence of free lunch, and introduces the concept of consistent bid-ask prices in a general setting with convex sets of marketed claims and convex pricing rules. Section 3 specializes to sets of marketed claims that are convex cones and to sublinear pricing rules. Section 4 studies the case where the opportunity set is the reduced form of a multiperiod securities market model and relates some of our results to independent work by Jacka (1992) and Ansel and Stricker (1994). Section 5 concludes. All proofs are contained in the appendix.

\section{The general model: convex pricing rules}

We denote by $R$ the real line and by $\bar{R}$ the extended real line $R \cup\{-\infty,+\infty\}$. If $x$ is a vector in $R^{d}$, where $d$ is an integer, we say that $x$ is nonnegative (resp. 
positive) and we write $x \geq 0$ (resp. $x>0$ ), if all the coordinates of $x$ are nonnegative (resp. nonnegative and at least one of them is positive). Let $(\Omega, \mathcal{F}, P)$ be a probability space and let $X=L^{p}\left(\Omega, \mathcal{F}, P ; R^{d}\right)$, with $1 \leq p \leq \infty$, be the space of all (equivalence classes of) $R^{d}$-valued and $\mathcal{F}$-measurable integrable random variables equipped with the classical $L^{p}$-norm if $1 \leq p<\infty$ and with the $\sigma\left(L^{\infty}, L^{1}\right)$, also called weak-* topology, if $p=\infty$. It is well known that $X$ is then a complete topological vector space. Let $X_{+}$be the set of elements $x$ of $X$ such that $P(x \geq 0)=1$ and $P(x>0)>0$. If $S$ is a subset of $X$, we denote by $\bar{S}$ the closure of $S$, and by cone $(S)$ the convex cone generated by $S$. A functional $f: X \rightarrow R$ is said to be positive if for all $x \in X_{+}$we have $f(x)>0$. We denote the set of positive linear functionals on $X$ by $\Psi$. We shall denote by $\operatorname{Im}(f)$ the range of $f$, i.e. the set $\{f(x): x \in X\}$. Note that the set of continuous linear functionals on $X$ can be identified with $L^{q}\left(\Omega, \mathcal{F}, P ; R^{d}\right)$ where $q$ is defined by the relation $\frac{1}{p}+\frac{1}{q}=1$ (with, by convention $q=1$ if $p=\infty$ ), and $\Psi$ can be identified with $L_{+}^{q}\left(\Omega, \mathcal{F}, P ; R^{d}\right)$.

Consider a two period economy where agents consume at the initial and the final date only and where they can purchase, at the initial date, contingent claims $m$ to consumption at the final date that belong to the set of marketed claims $M$, for a price $\pi(m)$ assigned by the pricing rule $\pi$ in units of consumption at the initial date. The opportunity set in this economy is therefore entirely described by the price system $(M, \pi)$. Furthermore, we impose

\section{Assumption 2.1 :}

(i) The set of marketed contingent claims $M$ is a convex ${ }^{3}$ subset of $X$ containing 0 ,

(ii) The pricing rule $\pi$ is a lower semicontinuous (l.s.c. $)^{4}$ real valued convex ${ }^{5}$ function defined on $M$ satisfying $\pi(0)=0$.

(iii) The constant function 1 equal to 1 almost everywhere belongs to $M$ and $\pi(\mathbf{1})=1$.

The fact that the set of marketed claims $M$ is convex means that if two claims are marketed, their average is marketed as well, and the fact that the pricing rule $\pi$ is convex means that its price is lower than or equal to their average

\footnotetext{
${ }^{3} \mathrm{~A}$ subset $M$ of $X$ is said to be convex if for all $x, y$ in $M$ and all $\lambda$ in $[0,1]$ we have $\lambda x+(1-\lambda) y \in M$.

${ }^{4} \mathrm{~A}$ function $\pi^{\prime}$ is said to be 1.s.c. if the set $\left\{(m, \lambda) \in M \times R: \lambda \geq \pi^{\prime}(m)\right\}$ is closed in $M \times R$, or equivalently if the set $\left\{m \in M: \lambda \geq \pi^{\prime}(m)\right\}$ is closed in $M$ for all $\lambda \in R$, or finally (in $L^{p}$ with $1 \leq p<\infty)$ if $\liminf _{n}\left\{\pi^{\prime}\left(m_{n}\right)\right\} \geq \pi^{\prime}(m)$ whenever the sequence $\left(m_{n}\right) \subset M$ converges to $m \in M$.

${ }^{5}$ A functional $\pi: M \rightarrow R$ defined on the convex set of marketed contingent claims $M$, is said to be convex if for all $x, y$ in $M$ and all $\lambda$ in $[0,1]$ we have $\pi(\lambda x+(1-\lambda) y) \leq \lambda \pi(x)+(1-\lambda) \pi(y)$.
} 
price. The fact that $M$ contains 0 and that $\pi(0)=0$ means that agents may keep their endowment and not purchase any other contingent claim, at a zero cost. Furthermore, this implies that the price at which a contingent claim can be purchased is larger than or equal to the price at which it can be sold (indeed $0=\pi(0)=\pi\left(\frac{1}{2} m+\frac{1}{2}(-m)\right) \leq \frac{1}{2} \pi(m)+\frac{1}{2} \pi(-m)$ implies $-\pi(-m) \leq \pi(m)$ for any marketed contingent claim $m$ ). The lower semicontinuity of the pricing rule $\pi$ is a technical assumption (which will not be needed at all in section 2), which basically means that there is no way of getting arbitrarily close to a contingent claim at a strictly lower cost. The fact that the constant function equal to 1 almost everywhere is marketed means that agents can acquire riskless contingent claims to consumption at the final date. The fact that $\pi(\mathbf{1})=1$ means that the riskless rate is equal to zero, and is merely a normalization which can be made without loss of generality as long as there exists a strictly positive contingent claim $m_{0}$ in $M$ by which all prices and payoffs can be normalized.

The fact that there are only two periods in this economy is not really a restriction since the price system $(M, \pi)$ can be viewed as the reduced form of a multiperiod securities market model where agents use dynamic trading strategies in order to transfer consumption from the initial to the final date, and are subject to a wide range of frictions such as portfolio constraints and trading fees or other costs. For instance, if markets are frictionless the resulting set of marketed claims $M$ is a linear subspace of $X$ and the pricing rule $\pi$ is linear (see Harrison and Kreps [1979]). For a large class of market frictions - including bid-ask spreads, shortselling constraints and costs, borrowing constraints and costs, and taxes - the resulting set of marketed claims $M$ is a convex cone and the pricing rule $\pi$ is sublinear and hence convex (see Jouini and Kallal [1995 a and b]). ${ }^{6}$ However, in a situation where transaction costs increase less than proportionally with transaction size, the set of marketed claims $M$ is still a convex cone and the pricing rule $\pi$ is convex (but not sublinear). In a situation where agents have risk limits on their portfolio, the set of marketed claims $M$ is of the form $M=\left\{m \in X: E\left(m^{2}\right)-E(m)^{2} \leq s^{2}\right\}$, where $s$ is the maximum amount of standard deviation allowed. In this case $M$ is convex (but is not a cone) and the pricing rule $\pi$ is convex (but is not sublinear).

The consumption space in this economy is hence represented by $R \times X$, and we assume that every agent is defined by a preorder of preferences $\preceq$ satisfying

\section{Assumption 2.2 :}

(i) for all $\left(r^{*}, x^{*}\right) \in R \times X,\left\{(r, x) \in R \times X:\left(r^{*}, x^{*}\right) \preceq(r, x)\right\}$ is convex,

(ii) for all $\left(r^{*}, x^{*}\right) \in R \times X,\left\{(r, x) \in R \times X:\left(r^{*}, x^{*}\right) \preceq(r, x)\right\}$ and $\{(r, x) \in R$ $\left.\times X:(r, x) \preceq\left(r^{*}, x^{*}\right)\right\}$ are closed, and

\footnotetext{
${ }^{6}$ Chen [1992] analyzes economies with taxes.
} 
(iii) for all $\left(r^{*}, x^{*}\right) \in R \times X$, for all $r>0$ and all $x \in X_{+}$we have $\left(r^{*}, x^{*}\right) \prec$ $\left(r^{*}+r, x^{*}\right)$ and $\left(r^{*}, x^{*}\right) \prec\left(r^{*}, x^{*}+x\right)$.

This means that preferences are assumed to be $(i)$ convex, (ii) continuous and (iii) strictly monotonic. This class of preferences is standard in equilibrium theory (see Debreu [1959]), and is analogous to the class used by Harrison and Kreps (1979).

We shall now define the price systems $(M, \pi)$ that are viable as models of economic equilibrium for agents with preferences satisfying Assumption 2.2. We shall follow closely the definition of viability introduced by Harrison and Kreps (1979), except that because of the frictions modeled by the convexity of the pricing rule and of the set of marketed claims, our agents have budget sets that are convex sets instead of being half linear spaces.

Definition 2.1 : The price system $(M, \pi)$, where the set of marketed contingent claims $M$ is a convex subset of $X$ and $\pi$ is a convex pricing rule defined on $M$, is said to be viable if there exists a preorder $\preceq$ satisfying Assumption 2.2 and such that $(r, m) \preceq(0,0)$ for all $(r, m) \in R \times M$ satisfying $r+\pi(m) \leq 0$.

This means that a viable price system $(M, \pi)$ is one for which we can find some agents with strictly monotonic, convex and continuous preferences, who can trade claims in $R \times M$ at prices given by the pricing rule $\pi$, and who are happy with their endowments, i.e. for whom supply is equal to demand. This means that viability is the minimal requirement for a price system $(M, \pi)$ to model an economic equilibrium, i.e. an economy where maximizing agents take prices as given and where supply is equal to demand. We then have

Theorem 2.1 : The price system $(M, \pi)$, where the set of marketed contingent claims $M$ is a convex subset of $X$ and $\pi$ is a convex pricing rule defined on $M$, is viable if and only if there exists a strictly positive and continuous linear functional $\psi$ defined on $X$ such that

$$
\left.\psi\right|_{M} \leq \pi
$$

This means that $(M, \pi)$ is viable if and only if there exists a linear pricing rule that is viable (when associated to the set of marketed claims $M$ ) and lies below $\pi$ on $M$. We shall call such a linear pricing rule an underlying frictionless pricing rule. Note that a necessary, although not sufficient, condition of viability is that $\pi$ is positive. Also note that if $M$ is a linear subspace of $X$ and $\pi$ is a linear pricing rule as in the frictionless case, we find the classical result of Harrison and Kreps (1979): $(M, \pi)$ is viable if and only if there exists a strictly positive linear extension of $\pi$ to the whole space $X$.

In some of the multiperiod securities price models with market frictions of which $(M, \pi)$ is a reduced form, the underlying frictionless pricing rules can be identified with the martingale, supermartingale, or submartingale measures of the 
adequately normalized securities price processes (or of some related processes). For instance, it is well known that if markets are incomplete (but otherwise frictionless) - under suitable assumptions - the underlying frictionless pricing rules are the martingale measures of the securities price processes normalized by a numeraire (see Harrison and Kreps [1979] and Delbaen and Schachermayer [1994b]). In securities markets with bid-ask spreads, they are the martingale measures of the processes that lie between the normalized bid and ask price processes of the traded securities (see Jouini and Kallal [1995 a]). In securities markets with short sales constraints, they are the supermatingale measures of the normalized traded securities price processes (see Jouini and Kallal [1995 b]).

We shall also relate the viability of a price system to the absence of arbitrage, or more precisely to the absence of free-lunch as defined in Delbaen and Schachermayer (1994a) and in Stricker (1990). In fact, in order to obtain a necessary and sufficient condition for the viability of a price system we have to consider a slightly more general concept: asymptotic free-lunches. ${ }^{7}$

Definition 2.2 : There is no asymptotic free-lunch if $\overline{\text { cone }}\left(C-(R \times X)_{+}\right) \cap$ $(R \times X)_{+}=\emptyset$, where $C=\{(r, m) \in R \times M: r+\pi(m) \leq 0\}$, and $(R \times X)_{+}=$ $\left\{(r, x) \in R \times X: r \geq 0, x \geq 0\right.$ and $\left.r+x \in X_{+}\right\}$.

This means that an asymptotic free-lunch is a way of getting arbitrarily close to a positive payoff at a nonnegative cost. Note that the no free-lunch property is usually defined by a condition on the space of contingent claims $X$ instead of our consumption space $R \times X$. However, the two definitions are equivalent if any amount of consumption can be transfered from the initial to the final date, e.g. if $M$ is a cone (we only assume that $M$ is a convex set and even if $(r, m)$ satisfies the budget constraint, $(0, r+m)$ is not necessarily feasible). Hence, it is easy to show that this concept of asymptotic free-lunch is equivalent to the concept of free lunch as defined in Delbaen and Schachermayer (1994a) and in Stricker (1990) whenever $M$ is a convex cone ${ }^{8}$ instead of being merely a convex set. Also, if $M-X_{+} \subset M$, which means that there is free-disposal in this economy, and if $\pi$ is nondecreasing, then the absence of asymptotic free-lunch is equivalent to the simpler condition $\overline{c o n e}(C) \cap(R \times X)_{+}=\emptyset$. We then have

Theorem 2.2 : The price system $(M, \pi)$, where the set of marketed contingent claims $M$ is a convex subset of $X$ and $\pi$ is a convex pricing rule defined on $M$, admits no asymptotic free-lunch if and only if it is viable.

\footnotetext{
${ }^{7}$ Recall that if $S$ is a subset of $X$, we denote by $\overline{c o n e}(S)$ the closure of the convex cone generated by $S$, where the closure is the strong closure if $p \geq 1$, and the weak-* closures if $p=\infty$ (as in Delbaen and Schachermayer [1994a]).

${ }^{8} \mathrm{~A}$ subset $M$ of $X$ is said to be a convex cone if it is convex and if for every $x$ in $M$ and every real number $\lambda \geq 0$, we have $\lambda x \in M$.
} 
We shall now consider contingent claims $x$ that belong to the consumption space $X$ but do not necessarily belong to the set of marketed claims $M$, and we shall examine what prices would be reasonable for the claim $x$ in the sense of viability. To do this, we introduce the notion of bid-ask prices consistent with the price system $(M, \pi)$. We denote by $M_{x}$ the convex hull of $M \cup\{-x, x\}$, which represents the extended set of marketed claims. By definition, $M_{x}$ is the smallest convex set containing $M,-x$ and $x$. Note that the claims that belong to $M_{x}$ are those which can be written as $(1-\lambda) m+\lambda x$ or $(1-\lambda) m-\lambda x$, for some $m \in M$ and $\lambda \in[0,1]$.

Definition 2.3 : Let $(M, \pi)$ be a viable price system, where the set of marketed contingent claims $M$ is a convex subset of $X$ and $\pi$ is a convex pricing rule defined on $M$. Let $x \in X$ and $(Q, P) \in \bar{R}^{2}$, we say that $(Q, P)$ is a bid-ask price pair for $x$ consistent with $(M, \pi)$ if there exists a l.s.c. convex functional $\pi^{\prime}$ defined on the extended convex set of marketed claims $M_{x}$, the convex hull of $M \cup\{-x, x\}$, satisfying $\pi^{\prime}(0)=0$ and such that

(i) $\left(-\pi^{\prime}(-x), \pi^{\prime}(x)\right)=(Q, P)$,

(ii) $\left.\pi^{\prime}\right|_{M} \leq \pi$, and

(iii) $\left(M_{x}, \pi^{\prime}\right)$ is viable. ${ }^{9}$

This means that a bid-ask price pair for $x$ is consistent with the viable price system $(M, \pi)$ if there exists at least a viable extended economy $\left(M_{x}, \pi^{\prime}\right)$ such that $(i)$ this pair is the actual bid-ask price pair for the contingent claim $x,(i i)$ equilibrium prices are within the original equilibrium bid-ask prices for every claim that belongs to $M$, and (iii) the equilibrium allocations are unchanged (i.e. agents are still happy with their initial endowments, given the new investment opportunities, and the possibly tighter bid-ask prices in the market). A typical objection is that when a contingent claim $x$ is added to the set of marketed claims (and as the bid-ask spreads for the originally marketed claims are potentially made tighter), agents may reshuffle their portfolios and as a result the entire price system may have to change to preserve equilibrium between supply and demand. However, it is reasonable to ask which prices are consistent with the equilibrium price system in the sense that they would not necessarily make the equilibrium collapse. In other words, consistency of a bid-ask price pair is a minimal requirement for this pair to be observed in equilibrium in at least an economy $(M, \pi)$ where the market for a new contingent claim opens. We then have

\footnotetext{
${ }^{9}$ In fact, to be consistent with our framework we should not allow for infinite prices. The extension to this case, however, is straightforwood. In particular, the viability of $\left(M_{x}, \pi^{\prime}\right)$ implies that $P>-\infty$ and $Q<+\infty$. If $P=+\infty$ this means that the claim $x$ is not marketed and if $Q=-\infty$ it means that a short position in the claim $x$ is not marketed.
} 
Theorem 2.3 : If $(M, \pi)$ is a viable price system, where the set of marketed contingent claims $M$ is a convex subset of $X$ and $\pi$ is a convex pricing rule defined on $M$, then for any $x \in X$ there exists some bid-ask price pair $(Q, P)$ that is consistent with $(M, \pi)$, and the pair $(Q, P)$ can be chosen such that $Q=P$ is finite. Furthermore, any price of the form $\psi(x)$, where $\psi \in \Psi$ and $\left.\psi\right|_{M} \leq \pi$ is a (bid or ask) price for $x$ consistent with $(M, \pi) .{ }^{10}$

Note that it does not mean that there will be no bid-ask spread for the contingent claim $x$ when this market opens; it means instead that there is no bid-ask spread for $x$ in at least an extended economy where bid-ask prices are overall at least as tight as in the original economy, and where equilibrium allocations remain unchanged. Furthermore, we have

Theorem 2.4 : If $(M, \pi)$ is a viable price system, where the set of marketed contingent claims $M$ is a convex subset of $X$ and $\pi$ is a convex pricing rule defined on $M$, then if $f^{11} 1 \leq p<\infty$ and if we define $\pi_{r}(y)$ for all $y \in X$ by

$$
\pi_{r}(y)=\inf \left\{\liminf _{n}\left\{\pi\left(m_{n}\right):\left(m_{n}\right) \subset M \text { and converges to } y\right\}\right.
$$

(i) The pair $\left(-\pi_{r}(-x), \pi_{r}(x)\right)$, is a bid-ask price pair for $x$ consistent with $(M, \pi)$.

(ii) Any consistent bid or ask price of $x$ lies between $-\pi_{r}(-x)$ and $\pi_{r}(x)$.

(iii) The pricing rule $\pi_{r}$ is the largest l.s.c. pricing rule that is lower than or equal to $\pi$.

Note that $\pi_{r}$ is lower than or equal to the pricing rule $\pi$ since it represents the minimum cost of getting arbitrarily close to a contingent claim.

\section{Sublinear pricing rules}

In order to analyze further the links between consistent prices and underlying frictionless economies, we have to impose

\section{Assumption 3.1 :}

(i) The set of marketed contingent claims $M$ is a convex cone, i.e. a convex set such that for all $x \in M$ and all nonnegative real number $\lambda$ we have $\lambda x \in M$.

(ii) The l.s.c. pricing rule $\pi$ is sublinear, ${ }^{12}$ i.e. is convex and such that for all $x \in M$ and all nonnegative real number $\lambda$ we have $\pi(\lambda x)=\lambda \pi(x)$.

\footnotetext{
${ }^{10}$ Note that if $(Q, P)$ is a bid-ask price pair for $x$ consistent with $(M, \pi)$ then $P \geq Q$.

${ }^{11}$ If $p=\infty$, we can state an analogous result but we have to replace the sequences by nets.

${ }^{12}$ Note that a convex pricing rule such that returns increase with the size of the transaction (i.e. $\pi(\lambda x) \leq \lambda \pi(x))$ is sublinear.
} 
The fact that $M$ is a cone (in addition to being convex) means that a marketed claim can be purchased in any arbitrary amount. The fact that the pricing rule $\pi$ is sublinear (in addition to being convex) means that the price of a marketed claim is proportional to the quantity of the claim purchased. As we have already mentioned, this is the case of the reduced form $(M, \pi)$ of multiperiod securities markets with a wide range of frictions including taxes, bid-ask spreads, shortselling constraints and costs, and borrowing constraints and costs (see Jouini and Kallal [1995 a and b]). In this context, the definition of consistent prices needs to be modified slightly as follows

Definition 3.1 : Let $(M, \pi)$ be a viable price system, where the set of marketed contingent claims $M$ is a convex cone of $X$ and $\pi$ is a convex sublinear pricing rule defined on $M$. Let $x \in X$ and $(Q, P) \in \bar{R}^{2}$, we say that $(Q, P)$ is a bid-ask price pair for $x$ consistent with $(M, \pi)$ if there exists a l.s.c. sublinear functional $\pi^{\prime}$ defined on the convex cone $M+R x$ such that

(i) $\left(-\pi^{\prime}(-x), \pi^{\prime}(x)\right)=(Q, P)$,

(ii) $\left.\pi^{\prime}\right|_{M} \leq \pi$, and

(iii) $\left(M+R x, \pi^{\prime}\right)$ is viable.

Note that this definition is identical to Definition 2.3 except that we have replaced the convex set $M_{x}$, the convex hull of $M \cup\{-x, x\}$, by the convex cone $M+R x$ to reflect the fact that there are no constraints on transaction sizes in this economy. In this context, we have the following result, which is a direct consequence of Theorem 2.2.

\section{Theorem 3.1 :}

(i) The price system $(M, \pi)$, where the set of marketed contingent claims $M$ is a convex cone of $X$ and $\pi$ is a convex sublinear pricing rule defined on $M$, is viable if and only if $\overline{\{x \in M: \pi(x) \leq 0\}-X_{+}} \cap X_{+}=\emptyset$ (no free-lunch condition).

(ii) If $M-X_{+} \subset M$ (i.e. if there is free disposal) and if $\pi$ is nondecreasing then the price system $(M, \pi)$ is viable if and only if $\{x \in M: \pi(x) \leq 0\} \cap X_{+}=\emptyset$ (no-arbitrage condition)

Note that the no-arbitrage condition is a minimum requirement of any reasonable model since it only expresses the fact that a positive payoff cannot be obtained for a nonpositive price. This Theorem states that this condition is equivalent to the viability of a price system under the assumption that the pricing rule is nondecreasing and that there is free-disposal in the set of marketed claims (which is the case for most reduced form multiperiod securities markets models). More generally, the Theorem states that the viability of a price system is equivalent to the absence of free lunch, , as defined in Delbaen and Schachermayer (1994a) and Stricker (1990), a weaker form of arbitrage opportunity. To proceed, we shall 
need

Definition 3.2 : $\quad$ Let $(M, \pi)$ be a viable price system, where the set of marketed contingent claims $M$ is a convex cone of $X$ and $\pi$ is a convex sublinear pricing rule defined on $M$, and let $x \in X$. We define by

(i) $C(x)=\left\{(Q, P) \in \bar{R}^{2}:(Q, P)\right.$ is a bid-ask price pair for $x$ consistent with $(M, \pi)\}$ the set of consistent bid-ask price pairs of $x$, and

(ii) $\Pi(x)=\left\{\psi(x): \psi \in \Psi\right.$ and $\left.\left.\psi\right|_{M} \leq \pi\right\}$ the set of underlying frictionless prices of $x$.

Note that $\Pi(x)$ is the set of prices of $x$ for all the underlying frictionless linear pricing rules and it is easy to show that $\Pi(x)=-\Pi(-x)$. Also note that if $(M, \pi)$ is a viable price system then $\Pi(x)$ is a nonempty interval of $R$, since the set $\left\{\psi \in \Psi:\left.\psi\right|_{M} \leq \pi\right\}$ is nonempty and convex. We then have

Theorem 3.2 : If $(M, \pi)$ is a viable price system, where the set of marketed contingent claims $M$ is a convex cone of $X$ and $\pi$ is a convex sublinear pricing rule defined on $M$, then for all $x \in X$,

(i) $C(x)$ is closed,

(ii) $\overline{(\Pi(x) \times \Pi(x)) \cap\{(Q, P): P \geq Q)\}} \subset C(x)$, and $C(x)$

(iii) If $-X_{+} \subset M$ and $\pi(-y) \leq 0$ for all $y \in X_{+}$, then $\overline{(\Pi(x) \times \Pi(x)) \cap\{(Q, P): P \geq Q)\}}=$

This means that if negative contingent claims are marketed at a nonpositive price in the economy, which is typically the case for reduced forms of multiperiod securities market models (to see this, consider the null strategy), the set of consistent prices of a contingent claim is a closed interval and is equal to (the closure of) the set of its underlying frictionless prices. However, in general the set $C(x)$ is not exactly equal to the set $(\Pi(x) \times \Pi(x)) \cap\{(Q, P): P \geq Q\}$ : indeed, it is easy to construct examples for which $\pi(-x)=0$ for some $x \in X_{+}$and such that $(M, \pi)$ is viable. ${ }^{13}$ In section 4 we shall study in more detail the boundary of the set $(\Pi(x) \times \Pi(x)) \cap\{(Q, P): P \geq Q\}$, and more specifically we shall characterize the situations where the set $\Pi(x)$ is closed.

Note that in the particular case where the contingent claim $x$ belongs to the set of marketed claims $M$, we have the equality $C(x)=([-\pi(-x), \pi(x)] \times$ $[-\pi(-x), \pi(x)]) \cap\{(Q, P): P \geq Q\}$. If $(M, \pi)$ is the reduced form of a multiperiod securities price model, then $\pi(x)$ is the minimum amount it costs to obtain the claim $x$, and $-\pi(-x)$ is the maximum amount that can be borrowed against it

\footnotetext{
${ }^{13}$ For instance, consider an economy where there are two dates and two states of the world at the final date, with only one security: a riskless asset. The resulting pricing rule $\pi$, which is defined by $\pi\left(x_{1}, x_{2}\right)=\max \left\{x_{1}, x_{2}\right\}$, is a viable pricing rule and is such that $\pi(0,-1)=0$, even though the contingent claim $(0,1)$ belongs to $X_{+}$.
} 
by trading securities. Hence, the claim $x$ would not be bought for more than $\pi(x)$ or sold for a price lower than $-\pi(-x)$ in any extended economy, since a better deal could be achieved through securities trading in both cases. This means that the interval $[-\pi(-x), \pi(x)]$ is the set of admissible prices for the claim $x$. Note that this does not mean that if the bid price of $x$ is below $-\pi(-x)$ or if the ask price of $x$ is above $\pi(x)$ then there are opportunities of arbitrage; it only means that there are no transactions at these prices. However, if the bid price of $x$ were to be above $\pi(x)$ or its ask price were to be below $-\pi(-x)$ then selling the claim $x$ at the bid price or buying it at the ask price and hedging the position through securities trading would constitute an arbitrage opportunity. Since we have $C(x)=\overline{(\Pi(x) \times \Pi(x)) \cap\{(Q, P): P \geq Q\}}$ by Theorem 3.1, this implies $[-\pi(-x), \pi(x)]=\overline{\Pi(x)}$. Also recall that Jouini and Kallal (1995 a and b) characterize the set $\Pi(x)$, and hence the interval of arbitrage bounds $[-\pi(-x), \pi(x)]$, using the set of martingale measures, supermartingale measures and submartingale measures, in various economies with bid-ask spreads, incomplete markets, short sales constraints, shortselling costs, and borrowing costs.

As we have already mentioned, a typical objection to the concept of consistent prices is that as the market for a non marketed contingent claim $x$ opens (and as bid-ask spreads in the originally marketed claims potentially tighten), new opportunities become available to agents and the old equilibrium might collapse. In a frictionless economy, this does not happen if the contingent claim $x$ is priced by arbitrage, i.e. if it admits a unique consistent price (see Kreps [1981, Theorem $5]$ ): in this case, even when the new claim $x$ is introduced, an equilibrium remains so. Similarly, in economies with market frictions we show that there exists a unique price system, namely the supremum of all the underlying frictionless pricing rules, for which equilibria do not collapse as the market for a new contingent claim $x$ opens (and as bid-ask spreads in the originally marketed claims potentially tighten). Indeed, we have

Theorem 3.3 : Let $(M, \pi)$ be a viable price system, where the set of marketed contingent claims $M$ is a convex cone of $X$ and $\pi$ is a convex sublinear pricing rule defined on $M$. Given a contingent claim $x \in X$, consider the extended set of marketed claims $M+R x$, and let the pricing rule $\pi^{\prime}$ be defined for all $m^{\prime} \in M+R x$ by

$$
\pi^{\prime}\left(m^{\prime}\right)=\sup _{\psi^{\prime} \in \Psi:\left.\psi^{\prime}\right|_{M} \leq \pi}\left\{\psi^{\prime}\left(m^{\prime}\right)\right\}
$$

(i) Then the price system $\left(M+R x, \pi^{\prime}\right)$ is viable, consistent with $(M, \pi)$, and such that $\left(r^{\prime}, m^{\prime}\right) \preceq(0,0)$ for all $\left(r^{\prime}, m^{\prime}\right) \in R \times(M+R x)$ such that $r^{\prime}+\pi^{\prime}\left(m^{\prime}\right) \leq$ 0 , for every preoder of preferences $\preceq$ satisfying Assumption 2.2 and such that $(r, m) \preceq(0,0)$ for all $(r, m) \in R \times M$ such that $r+\pi(m) \leq 0$.

(ii) Moreover, if $-X_{+} \subset M$ and $\pi(-y) \leq 0$ for all $y \in X_{+}$, then $\left(M+R x, \pi^{\prime}\right)$ 
is the unique l.s.c. price system satisfying (i).

Therefore, based on equilibrium or arbitrage considerations alone, and without knowledge about preferences and endowments, it is not possible to infer tighter bounds than the arbitrage bounds given by the interval

$$
\left[\inf _{\psi \in \Psi:\left.\psi\right|_{M} \leq \pi}\{\psi(x)\}, \sup _{\psi \in \Psi:\left.\psi\right|_{M} \leq \pi}\{\psi(x)\}\right]=\overline{\Pi(x)}
$$

on the bid-ask prices of a contingent claim $x$. Indeed, any tighter bid-ask price interval would lead to a collapse of the equilibrium in place, in at least an economy. ${ }^{14}$ Hence, $\inf _{\psi \in \Psi:\left.\psi\right|_{M} \leq \pi}\{\psi(x)\}$ and $\sup _{\psi \in \Psi:\left.\psi\right|_{M} \leq \pi}\{\psi(x)\}$ can be interpreted as the bid and the ask prices of the contingent claim $x \in X$.

\section{Pricing rules from trading strategies}

In this section, we shall assume that marketed contingent claims are available to agents as the result of trading strategies, as in multiperiod securities markets models (see for unstance Harrison and Kreps[1979] for the frictionless case and Jouini and Kallal [1995 a and b] for models with market frictions). More precisely, let $\Sigma$ be a convex cone in a topological vector space representing the space of admissible strategies and $c$ be a nondecreasing real-valued sublinear mapping on $\Sigma$. The convex cone $\Sigma$ represents the space of self-financing strategies (i.e. that do not require any infusion of funds other than at the initial date) and for any strategy $\sigma \in \Sigma, c(\sigma)$ represents the initial investment necessary to carry out the strategy $\sigma$. To each strategy $\sigma$, we associate its resulting payoff $R(\sigma)$ in $X$, where $R$ is a superlinear ${ }^{15}$ mapping between $\Sigma$ and $X$.

In this context, a pricing rule $P$ is defined as a l.s.c. sublinear functional such that $P(x)$, the price of a given contingent claim $x$, is lower than or equal to the minimum cost necessary to dominate $x$. Since $P$ is l.s.c., it is easy to see that

$$
P(x) \leq P^{*}(x) \equiv \inf \liminf _{R\left(\sigma_{n}\right) \geq x_{n}, x_{n} \rightarrow x}\left\{c\left(\sigma_{n}\right)\right\}
$$

the minimum cost necessary to dominate $x$ or to dominate some payoffs arbitrarily close to $x$. Moreover, if $p<\infty$ then $P^{*}$ is the greatest 1.s.c. sublinear functional such that the price every contingent claim $x$ is lower than or equal to the minimum

\footnotetext{
${ }^{14}$ Unfortunately, these bounds can be quite wide, as shown for instance by Soner, Shreve and Cvitanic (1995) in a model where the risky asset follows a geometric brownian motion and is subject to proportional transaction costs. By imposing restrictions on preferences and/or endowments Davis et al. (1993) and Constantinides (1993), among others, obtain tighter bounds.

${ }^{15}$ This means that $R\left(\sigma_{1}+\sigma_{2}\right) \geq R\left(\sigma_{1}\right)+R\left(\sigma_{2}\right)$ for all $\sigma_{1}, \sigma_{2} \in \Sigma$.
} 
cost necessary to dominate it. ${ }^{16}$ However, if $p=\infty$ Delbaen and Schachermayer (1994a) pointed out that the closure of a convex set cannot necessarily be obtained by taking limits of all sequences. Therefore, $P^{*}$ is not necessarily l.s.c. in this case.

As shown in the previous section (Theorem 3.1 and its consequences), if the price system is viable there exists some convex subset ${ }^{17} K \subset \Psi$ such that $P(x) \geq$ $\sup _{\psi \in K}\{\psi(x)\}$ for all $x \in X$, where the equality holds if $P(-y)<0$ for all $y \in X_{+}$. Analogously, if $P^{*}$ is viable, there exists $\psi \in \Psi$ such that ${ }^{18} P^{*}(x) \geq \psi(x)$ for all $x \in X$. In what follows, we shall denote by $K^{*}$ the set of all such linear functionals $\psi \in \Psi$ and we then have ${ }^{19} P^{*}(x) \geq \sup _{\psi \in K^{*}} \psi(x)$, where the equality holds if $p<\infty$ and if $P^{*}(-y)<0$ for all $y \in X_{+}$. As a particular case, if $\Sigma$ is a convex cone of $X$ denoted by $M, R$ the canonical injection, and if $c$ is denoted by $\pi$ we obtain the model of section 2 and $P^{*}$ is then the mapping $\pi_{r}$ defined in Theorem 2.4. We then have ${ }^{20}$

Theorem 4.1 : If $p>1$, then for every $x \in X$, if there exists $\psi_{0} \in K^{*}$ such that $P^{*}(x)=\psi_{0}(x)$ then $x \in \overline{\mathrm{Im}(R)}$

Note that the converse implication is not true in general. Indeed, consider a securities market model with two assets $(B$ and $S)$ and two states of the world $(u$ and $d$ ). The asset $B$ is a bond and has a constant price equal to 1 , the asset $S$ is a stock and pays 110 (resp. 90) units of consumption at date 1 in state $u$ (resp. d). We shall denote by $(110,90)$ this payoff. We assume that the stock can be bought at a price equal to 110 and sold at a price equal to 90 at date 0 . The space of strategies $\Sigma$ is equal to $R^{2}$ and an element $(\alpha, \beta)$ of $\Sigma$ represents a portfolio composed by $\alpha$ bonds and $\beta$ stocks. We then have $c(\alpha, \beta)=\alpha+110 \beta^{+}-90 \beta^{-}$ and $R(\alpha, \beta)=(\alpha+110 \beta, \alpha+90 \beta)$. In this framework, it is easy to see that $K^{*}=\{(p, 1-p): p \in] 0,1[\}, P^{*}(110,90)=110$ and that there is no $\psi$ in $K^{*}$ such that $P^{*}(110,90)=\psi(110,90)$.

In general frictionless but not necessarily complete markets, the space $\Sigma$ of admissible strategies is a vector space and the "cost mapping" $c$ and the "Result

\footnotetext{
${ }^{16}$ Indeed, $P^{*}$ is l.s.c. by construction and we have $P^{*}(x) \leq \inf _{R(\sigma)>x}\{c(\sigma)\}$. Moreover, $P(x) \leq \inf _{R(\sigma) \geq x}\{c(\sigma)\}$ and since $P$ is l.s.c. we have $P(x) \leq \operatorname{infliminf}_{y_{n} \rightarrow x}\left\{P\left(y_{n}\right)\right\}$ and since $P\left(y_{n}\right) \leq c\left(\sigma_{n}\right)$ whenever $y_{n} \leq R\left(\sigma_{n}\right)$ we obtain $P \leq P^{*}$.

${ }^{17}$ Indeed, to have the inequality it suffices to consider the set $K$ of underlying frictionless pricing rules $\psi \in \Psi$ such that $P \geq \psi$. If $P(-y)<0$ for all $y \in X_{+}$, then all such $\psi$ must satisfy $\psi(y)>0$ for all $y \in X_{+}$, and by Hahn-Banach Theorem and the fact that $P$ is l.s.c. we have the equality.

${ }^{18}$ This is a consequence of Theorem 2.1 where the lower semi-continuity of $\pi$ is not required in order to establish the result.

${ }^{19}$ Indeed, $P^{*}$ is l.s.c. if $p<\infty$.

${ }^{20}$ This Theorem relies on topological tools developed in Delbaen and Schachermayer (1994a), and in particular on a simple version of Komlos Theorem for the case where $p=\infty$.
} 
mapping" $R$ are both linear mappings. We then obtain as a simple consequence of our previous analysis a result also proved in Jacka (1992) as well as in Ansel and Stricker (1994).

Corollary 4.2 : In markets with no friction, for every $x \in X$ we have $P^{*}(x)=$ $\psi_{0}(x)$ for some $\psi_{0} \in K^{*}$ if and only if $\left\{\psi(x): \psi \in K^{*}\right\}$ is reduced to a unique element.

This means that if markets are incomplete but otherwise frictionless the set of underlying frictionless prices $\Pi(x)$ is closed (using the notation of section 3 ) if and only if it is a singleton.

\section{Conclusion}

In this paper, we have developed some foundational issues related to the theory of asset pricing, in securities markets with frictions. We model market frictions by letting the set of marketed contingent claims (i.e. the opportunity set) be a convex set and by letting the pricing rule at which these claims are available be convex. This is the reduced form of multiperiod securities price models, incorporating a large class of market frictions including bid-ask spreads, short sales and other portfolio constraints, shortselling costs, and borrowing costs. A price system is said to be viable as a model of economic equilibrium if there exist price-taking maximizing agents with a convex, continuous, and stricly increasing preorder of preferences who are happy with their endowments, and hence for whom supply is equal to demand. We show that a price system is viable if and only if there exists a positive linear pricing rule on the entire space of contingent claims - an underlying frictionless linear pricing rule - that lies below the convex pricing rule on the set of marketed claims. We also show that this is equivalent to the absence of asymptotic free lunches - a generalization of opportunities of arbitrage. When a market for a non marketed contingent claim opens, a bid-ask price pair is said to be consistent if it is a bid-ask price pair in at least a viable extended economy (where the new contingent claim can now be bought and sold) - with identical or tighter bid-ask spreads on the set of originally marketed claims. If the set of marketed contingent claims is a convex cone and if the pricing rule is sublinear, we show that the set of consistent prices of a contingent claim is a closed interval and is equal (up to its boundary) to the set of its prices for all the underlying frictionless pricing rules. As the market for a new contingent claim opens new opportunities arise, agents might reshuffle their portfolio, and the original equilibrium might collapse. However, we show that there exists a unique extended consistent sublinear pricing rule - the supremum of the underlying frictionless linear pricing rules - for which the original equilibrium does not collapse when a market for a contingent claim is opened, no 
matter what preferences and endowments are. This means that without restricting preferences and endowments, and based on arbitrage and equilibrium arguments alone, we cannot infer tighter bounds on the price of a contingent claim than the arbitrage bounds also given by the underlying frictionless pricing rules. We also study the closedness of the interval of underlying frictionless prices, in reduced forms of multiperiod securities prices models. In particular, in a model where markets are incomplete but otherwise frictionless we show that this interval is closed if and only if it is reduced to a single element (a result that can also be found in Jacka [1990] and Ansel and Stricker [1994]). 


\section{Appendix}

Proof of Theorem 2.1: Suppose that there exists a linear functional $\psi$ in $\Psi$ defined on $X$ such that $\left.\psi\right|_{M} \leq \pi$. Let $\preceq$ then be defined by $\left(r^{\prime}, x^{\prime}\right) \preceq(r, x)$ if $r^{\prime}+$ $\psi\left(x^{\prime}\right) \leq r+\psi(x)$. It is clear that $\preceq$ is an element of the class $\mathcal{C}$. Let $(r, m) \in R \times M$ such that $r+\pi(m) \leq 0$. We then must have $r+\psi(m) \leq 0$ since $\left.\psi\right|_{M} \leq \pi$. Noting that $0+\psi(0)=0$ we obtain that $(r, m) \preceq(0,0)$.

Conversely, suppose that $(M, \pi)$ is viable and hence let $\preceq$ be as in definition 2.1. Let $J=\{(r, x) \in R \times X:(0,0) \prec(r, x)\}$, and $K=\{(r, m) \in R \times$ $M: 0 \geq r+\pi(m)\}$. Then $J$ and $K$ are disjoint and convex; furthermore $J$ is open. By a separation theorem (see Jameson [1974, theorem 23.13]) there exists a nontrivial continuous linear functional $\phi$ on $R \times X$ and $\alpha \in r$ such that $\phi(r, x)>\alpha$, for all $(r, x) \in J$, and $\phi(r, m) \leq \alpha$, for all $(r, m) \in K$. Note that by continuity of $\phi$ and strict monotonicity of $\preceq$ we must have $\phi(0,0)=\alpha=0$. Moreover, by strict monotonicity of $\preceq$ we have $(0,0) \prec(1,0)$ and hence $(1,0) \in J$ which implies that $\phi(1,0)>0$. We then renormalize $\phi$ so that $\phi(1,0)=1$. We also let $\psi$ be defined on $X$ by $\psi(x)=\phi(0, x)$. It is easy to show that $\psi$ is a continuous and linear functional. If we let $x \in X_{+}$, we have by strict monotonicity of $\preceq$, $(0,0) \prec(0, x)$ and hence $(0, x) \in J$. Therefore we must have $\psi(x)>0$ and $\psi$ is positive. It only remains to prove that $\left.\psi\right|_{M} \leq \pi$. For this, let $m \in M$. It is clear that $(-\pi(m), m) \in K$. Consequently, $\phi(-\pi(m), m) \leq 0$ and $\psi(m)-\pi(m) \leq 0$. Thus, we must have $\left.\psi\right|_{M} \leq \pi$.

Proof of Theorem 2.2: Let $(M, \pi)$ be viable and hence such that there exists a positive linear functional $\psi \in \Psi$ such that $\left.\psi\right|_{M} \leq \pi$. Consider an element $(r, x) \in C$, we must have $r+\pi(x) \leq 0$ and then $r+\psi(x) \leq 0$. The positive linear functional $(r, x) \rightarrow r+\psi(x)$ is then nonpositive on $\overline{c o n e}\left(C-(R \times X)_{+}\right)$ and positive on $(R \times X)_{+}$. This implies the absence of asymptotic free-lunch.

Conversely, if $\overline{c o n e}\left(C-(R \times X)_{+}\right) \cap(R \times X)_{+}=\emptyset$, since $\overline{c o n e}\left(C-(R \times X)_{+}\right)$ and $(R \times X)_{+}$are cones (with vertices at the origin), with empty intersection, we then have according to the Kreps-Yan theorem ${ }^{21}$ (see, for example Delbaen and Schachermayer [1994a], proof of Theorem 1.1) that there exists a continuous linear functional $\bar{\phi}$ on $R \times X$ such that $\bar{\phi}((r, x))>0$ for all $(r, x) \in(R \times X)_{+}$and $\bar{\phi}((r, x)) \leq 0$ for all $(r, x) \in \overline{\text { cone }}\left(C-(R \times X)_{+}\right)$. We can then renormalize $\bar{\phi}$ in order to obtain $\phi((r, x))=r+\psi(x)$ satisfying the same properties, with $\psi \in \Psi$.

\footnotetext{
${ }^{21}$ In fact, for $1<p<\infty$, the Clark's (1993) separation theorem is sufficient. The theorem of Kreps-Yan is then usefull for $p=1$ or $p=\infty$. Note that we are applying these separation theorems in the space $R \times X$ which can be identified with $L^{p}(\widetilde{\Omega}, \widetilde{\mathcal{F}}, \widetilde{P})$ where $\widetilde{\Omega}=\Omega \cup\{\varpi\}$, $\widetilde{\mathcal{F}}=\mathcal{F} \cup\{\mathcal{A} \cup\{\varpi\}: \mathcal{A} \in \mathcal{F}\}$ and where $\widetilde{P}$ is defined, for all $A \in \mathcal{F}$ by $\widetilde{P}(A)=\frac{1}{2} \mathrm{P}(A)$ and $\widetilde{P}(A \cup\{\varpi\})=\frac{1}{2}+\frac{1}{2} \mathrm{P}(A)$.
} 
Let $m \in M$, we then have $(-\pi(m), m) \in \overline{c o n e}\left(C-(R \times X)_{+}\right)$which implies that $-\pi(m)+\psi(m) \leq 0$. This means that $\left.\psi\right|_{M} \leq \pi$.

Proof of Theorem 2.3: $\quad$ Let $(M, \pi)$ be viable, and let $x \in X$. Let $\psi \in \Psi$ such that $\left.\psi\right|_{M} \leq \pi$, as guaranteed by Theorem 2.1. Then $(\psi(x), \psi(x))$ is a bidask price pair for $x$ that is consistent with $(M, \pi)$. Indeed, $\pi^{\prime}$ defined on $M_{x}$ by $\pi^{\prime}=\left.\psi\right|_{M_{x}}$ is a convex functional (since it is linear ), which satisfies $\left.\pi^{\prime}\right|_{M} \leq \pi$ since $\left.\pi^{\prime}\right|_{M}=\left.\psi\right|_{M}$.

Proof of Theorem 2.4: The pricing rule $\pi_{r}$ is l.s.c. by construction and is lower than or equal to $\pi$ since the sequences $m_{n}$ include the constant sequences. Moreover, it is the largest l.s.c. pricing rule that is lower than or equal to $\pi$. Indeed, if $\tilde{\pi}$ is l.s.c. and is lower than or equal to $\pi$ then $\tilde{\pi}(y) \leq \inf \left\{\liminf \left\{\tilde{\pi}\left(m_{n}\right)\right.\right.$ : $\left(m_{n}\right) \subset M$ and converges to $\left.y\right\}$ for all $y \in M$ and since we have $\tilde{\pi}\left(m_{n}\right) \leq \pi\left(m_{n}\right)$ we obtain $\tilde{\pi}(y) \leq \pi_{r}(y)$. Therefore any pair of bid-ask prices for $x$ consistent with $(M, \pi)$ must be included in the closed interval $\left[-\pi_{r}(-x), \pi_{r}(x)\right]$.

Proof of Theorem 3.1: If $(M, \pi)$ is viable, then we can apply the result of Theorem 2.2 and we have that $\overline{c o n e}\left(C-(R \times X)_{+}\right) \cap(R \times X)_{+}=\emptyset$. Since $C$ is now a cone, this last condition is equivalent to $\overline{\left(C-(R \times X)_{+}\right)} \cap(R \times X)_{+}=\emptyset$. If we take the intersection with $\{0\} \times X$ we then have $\{x \in M: \pi(x) \leq 0\}-X_{+} \cap X_{+}=\emptyset$.

Conversely, if $\overline{\{x \in M: \pi(x) \leq 0\}-X_{+}} \cap X_{+}=\emptyset$, then let $\left(r^{*}, m^{*}\right)$ in $\overline{\left(C-(R \times X)_{+}\right)} \cap$ $(R \times X)_{+}$, it is easy to see that $r^{*}+m^{*}$ is then in $\overline{\{x \in M: \pi(x) \leq 0\}-X_{+}} \cap X_{+}$ which is impossible. We then have that $\overline{c o n e}\left(C-(R \times X)_{+}\right) \cap(R \times X)_{+}=\emptyset$ and $(M, \pi)$ is viable according to Theorem 2.2.

Finally, if $M-X_{+} \subset M$ and if $\pi$ is nondecreasing, then $\{x \in M: \pi(x) \leq 0\}-$ $X_{+}=\{x \in M: \pi(x) \leq 0\}$ which is closed by the lower semi-continuity of $\pi$. The condition then becomes $\{x \in M: \pi(x) \leq 0\} \cap X_{+}=\emptyset$

Proof of Theorem 3.2: First, we shall prove that, for all $x \in X$, the set $C(x)$ is closed. Indeed, let $\left(Q_{n}, P_{n}\right)$ be a sequence in $C(x)$ that converges to some $(Q, P)$. There exists a sequence $\pi_{n}^{\prime}$ of sublinear functions defined on $M+R x$ such that $\left.\pi_{n}^{\prime}\right|_{M} \leq \pi, \pi_{n}^{\prime}(x)=P_{n}$ and $\pi_{n}^{\prime}(-x)=-Q_{n}$, for all $n$. Since the sequences $\left(P_{n}\right)$ and $\left(Q_{n}\right)$ converge, they are bounded respectively by some real numbers $\bar{P}$ and $\bar{Q}$. Let $y$ be in $M+R x$, there exists $m \in M$ and two real numbers $\alpha$ and $\beta$ in $R_{+}$such that $y=m+(\alpha-\beta) x$. We then have $\pi_{n}^{\prime}(y) \leq \pi(m)+\alpha P_{n}-\beta Q_{n} \leq$ $\pi(m)+\alpha \bar{P}+\beta \bar{Q}$ and analogously $\pi_{n}^{\prime}(-y) \leq \pi(-m)+\alpha \bar{P}+\beta \bar{Q}$. Since $\pi_{n}^{\prime}(0)=0$ and hence $-\pi_{n}^{\prime}(-y) \leq \pi_{n}^{\prime}(y)$, we have $\left|\pi_{n}^{\prime}(y)\right| \leq|\pi(m)|+|\pi(-m)|+\alpha \bar{P}+\beta \bar{Q}$. The sequence $\pi_{n}^{\prime}(y)$ is then bounded and we can define a function $\pi^{\prime}$ on $M+R x$ by $\pi^{\prime}=\sup _{n} \sup _{t>n} \pi_{t}^{\prime}$. It is easy to show that $\pi^{\prime}$ is well defined, sublinear and such that $\left.\pi^{\prime}\right|_{M} \leq \pi, \pi^{\prime}(x)=P$ and $\pi^{\prime}(-x)=-Q$. This proves that $(Q, P) \in C(x)$ and that $C(x)$ is closed.

The theorem is then a consequence of the following lemma. 
Lemma : Let $(M, \pi)$ be a viable price system.

(i) If $Q$ and $P$ are in $\overline{\Pi(x)}$ with $Q \leq P$ then $(Q, P) \in C(x)$

(ii) Conversely, assuming that $-X_{+} \subset M$ and that $\pi(-y) \leq 0$ for all $y \in X_{+}$, if $(Q, P) \in C(x)$, then $Q$ and $P$ are included in $\overline{\Pi(x)}$.

Proof of the Lemma: Since $C(x)$ is closed, it suffices to prove (i) when $Q$ and $P$ are in $\Pi(x)$. If $Q$ and $P$ are in $\Pi(x)$ with $Q<P$, then there exist functionals $\psi_{P}$ and $\psi_{Q}$ in $\Psi$ such that $\left.\psi_{P}\right|_{M} \leq \pi,\left.\psi_{Q}\right|_{M} \leq \pi, \psi_{P}(x)=P$ and $\psi_{Q}(x)=Q$. Then consider the functional $\pi^{\prime}$ defined for all $x^{\prime} \in M+R x$, with $x^{\prime}=m+\lambda x$ for some $(m, \lambda) \in M \times R_{+}$, by $\left.\pi^{\prime}\left(x^{\prime}\right)=\max \left[\psi_{P}\left(x^{\prime}\right), \psi_{Q}\left(x^{\prime}\right)\right]\right\}$. It is easy to check that $\pi^{\prime}$ is l.s.c. and sublinear and that $\left(M+R x, \pi^{\prime}\right)$ is a viable (since $\pi^{\prime} \geq \psi_{P}$ ) price system satisfying $\left.\pi^{\prime}\right|_{M} \leq \pi$, and hence that $(Q, P)$ is a bid-ask price pair of $x$ consistent with $(M, \pi)$.

If $P \in \Pi(x)$, then there exists $\psi \in \Psi$ such that $\left.\psi\right|_{M} \leq \pi$ and $\psi(x)=P$. Let now $\pi^{\prime}: M+R x \rightarrow R$ be defined for all $x^{\prime} \in M+R x$ with $x^{\prime}=m+\lambda x$ for some $(m, \lambda) \in M \times R_{+}$, by $\pi^{\prime}\left(x^{\prime}\right)=\psi\left(x^{\prime}\right)$. It is easy to see that $\pi^{\prime}$ is a sublinear functional and we have that $\left.\psi\right|_{M+R x} \leq \pi^{\prime}$. Consequently, $\left(M+R x, \pi^{\prime}\right)$ is viable and $(P, P)$ is then a bid-ask price pair for $x$ consistent with $(M, \pi)$.

Conversely, assume $-X_{+} \subset M$ and $\pi(-y) \leq 0$ for all $y \in X_{+}$and let $(Q, P)$, with $Q<P$, be a bid-ask price pair for $x$ consistent with $(M, \pi)$. Then there exists a convex functional $\pi^{\prime}$ on $M+R x$ such that $\pi^{\prime}(x)=P, \pi^{\prime}(-x)=-Q,\left.\pi^{\prime}\right|_{M} \leq \pi$ and $\left(M+R x, \pi^{\prime}\right)$ is viable. Let $A=\left\{\left(x^{\prime}, \lambda\right) \in M+R x \times R: \lambda \geq \pi^{\prime}\left(x^{\prime}\right)\right\}$, since $\pi^{\prime}$ is l.s.c. then $A$ is a closed convex set and for all $\varepsilon>0,(x, P-\varepsilon) \notin A$. By a strict separation Theorem and for some $\varepsilon>0$, there exists a continuous linear functional $\phi$ defined on $X \times R$ and $\alpha \in R$ such that $\phi(x, P-\varepsilon)<\alpha$ and $\phi\left(x^{\prime}, \lambda\right)>\alpha$ for all $\left(x^{\prime}, \lambda\right) \in A$. Then there exists a continuous linear functional $\varphi$ on $X$ and $\beta \in R$ such that $\phi(y, \lambda)=\varphi(y)+\beta \lambda$, for all $(y, \lambda) \in X \times R$ and we must have, for all $\left(x^{\prime}, \lambda\right) \in A, \varphi\left(x^{\prime}\right)+\beta \lambda>\alpha$ and $\varphi(x)+\beta(P-\varepsilon)<\alpha$. Noting that $(0,0) \in A$ and $(x, \lambda) \in A$ for all $\lambda \geq \pi^{\prime}(x)$ it is easy to see that $\beta>0$ and $\alpha \leq 0$. Furthermore, since $A$ is a cone we have, for all $\left(x^{\prime}, \lambda\right) \in A, \varphi\left(x^{\prime}\right)+\beta \lambda \geq 0$.

Let us consider the continuous linear functional $\xi$ defined by $\xi(y)=-\frac{1}{\beta} \varphi(y)$, for all $y \in X$. Since $\left(x^{\prime}, \pi^{\prime}\left(x^{\prime}\right)\right) \in A$ for all $x^{\prime} \in M+R x$ we clearly have, $\xi\left(x^{\prime}\right) \leq$ $\pi^{\prime}\left(x^{\prime}\right)$ for all $x^{\prime} \in M+R x$ and $\xi(x)>p-\varepsilon$. In particular, for all $y \in X_{+}$we must have $\xi(-y) \leq \pi^{\prime}(-y) \leq 0$ and hence, $\xi(y) \geq 0$. Let now $\psi^{\prime}$ be a linear positive functional such that $\left.\psi^{\prime}\right|_{M+R x} \leq \pi^{\prime}$ as guaranteed by the viability of $\left(M+R x, \pi^{\prime}\right)$ and by Theorem 2.1. Let us define for $\gamma \in(0,1)$ the linear functional $\psi$ by $\psi(y)=\gamma \psi^{\prime}(y)+(1-\gamma) \xi(y)$ for $y \in X$. We clearly have that $\psi \in \Psi$, $\left.\psi\right|_{M} \leq \pi$ and for $\gamma$ sufficiently small we also must have $P-\varepsilon \leq \psi(x) \leq P$. A symmetric argument gives the existence of $\bar{\psi} \in \Psi$ such that $\left.\bar{\psi}\right|_{M} \leq \pi$ and $-Q-\varepsilon \leq \bar{\psi}(-x) \leq-Q$ which implies that $Q \leq \bar{\psi}(x) \leq Q+\varepsilon$. Therefore, $P$ and 
$Q$ are in $\overline{\Pi(x)}$.

Now let $P$ such that $(P, P)$ is a bid-ask price of $x$ consistent with $(M, \pi)$, then there exists a l.s.c. convex functional $\pi^{\prime}$ defined on $M+R x$ such that $\left.\pi^{\prime}\right|_{M} \leq \pi$, $\pi^{\prime}(x)=-\pi^{\prime}(-x)=P$ and $\left(M+R x, \pi^{\prime}\right)$ is viable. By Theorem 2.2 there exists a positive continuous linear functional $\psi$ on $X$ such that $\left.\psi\right|_{M+R x} \leq \pi^{\prime}$. By linearity of $\psi$ we must have $\psi(x)=P$ and hence $P \in \Pi(x)$.

Proof of Theorem 3.3: To prove $(i)$ it suffices to separate the convex sets $J$ and $K$ as in the proof of Theorem 2.2.

To prove (ii), let $\pi$ " be a sublinear extension of $\pi$ to $M+R x$ such that $\pi$ " $\left.\right|_{M} \leq \pi$ and such that $\pi^{\prime \prime}(x)<\pi^{\prime}(x)$, where $\pi^{\prime}(x)=\sup _{\psi^{\prime} \in \Psi:\left.\psi^{\prime}\right|_{M} \leq \pi}\left\{\psi^{\prime}(x)\right\}$. Then there exists $\psi \in \Psi$ such that $\left.\psi\right|_{M} \leq \pi$ and $\pi "(x)<\psi(x)$. Hence, we can define the preorder of preferences $\preceq_{\psi} \in \mathcal{C}$ by $\left(r^{\prime}, y^{\prime}\right) \preceq_{\psi}(r, y)$ if $r^{\prime}+\psi\left(y^{\prime}\right) \leq r+\psi(y)$. Then let $r$ " be the real number that solves $r "+\pi "(x)=0$. It also must satisfy $r "+\psi(x)>0$, i.e. $(0,0) \prec_{\psi}(r ", x)$, since $\pi "(x)<\psi(x)$. Hence $(M+R x, \pi ")$ is not an equilibrium price system in an economy populated by agents with a preorder of preferences $\preceq_{\psi}$ although $(M, \pi)$ is.

Proof of Theorem 4.1 : Let $x \in X$ such that $P^{*}(x)=\psi_{0}(x)$ for some $\psi_{0}$ in $K^{*}$. Then there exist sequences $\left(\sigma_{n}\right)$ in $\Sigma,\left(x_{n}\right)$ in $X$ converging to $x$ and $\left(h_{n}\right)$ in $X_{+}$such that $R\left(\sigma_{n}\right)=x_{n}+h_{n}$ and $c\left(\sigma_{n}\right)$ converges to $\psi_{0}(x)$. Let $\lambda_{n}=1$ if $\left\|h_{n}\right\| \leq 1$ and $\lambda_{n}=\frac{1}{\left\|h_{n}\right\|}$ otherwise. If $p<\infty$, then $X$ is reflexive and $X_{++} \cap\{x \in X:\|x\| \leq 1\}$ is a compact set for the weak-topology, where $X_{++}$ denotes the subset of nonnegative elements of $X$. Therefore, we can assume that $\lambda_{n} h_{n}$ converges to some $h$ in $X_{++}$. We then have $R\left(\sigma_{n}\right)=x_{n}+\lambda_{n} h_{n}+\left(1-\lambda_{n}\right) h_{n}$ where $x_{n}+\lambda_{n} h_{n}$ converges to $x+h$ and $\left(1-\lambda_{n}\right) h_{n}$ is in $X_{++}$. We then have, by definition of $P^{*}, P^{*}(x+h) \leq \lim _{n \rightarrow \infty} c\left(\sigma_{n}\right)=P^{*}(x)$. Furthermore, $P^{*}(x+h) \geq$ $\psi_{0}(x+h)=P^{*}(x)+\psi_{0}(h)$, and $h$ must be equal to 0 which implies that $h_{n}$ converges to 0 and $R\left(\sigma_{n}\right)$ converges to $x$.

If $p=\infty$, the sequence $\left(h_{n}\right)$ is nonnegative and bounded. Following Delbaen and Schachermayer (1994a, Lemma A.1.1) there exists a sequence $\left(\widetilde{h_{n}}\right) \in$ $\operatorname{conv}\left(h_{n}, h_{n+1}, \ldots\right)$ converging almost surely (and therefore in the sense of the $\sigma\left(L^{\infty}, L^{1}\right)$ topology), to a finite nonnegative function $h$. Let us denote by $\left(\widetilde{x_{n}}\right)$ and $\left(\widetilde{\sigma_{n}}\right)$ the sequences obtained by convex combination from $\left(x_{n}\right)$ and $\left(\sigma_{n}\right)$ with the same weights as $\left(\widetilde{h_{n}}\right)$. It is easy to see that $\left(\widetilde{x_{n}}\right)$ converges to $x, R\left(\widetilde{\sigma_{n}}\right)=\widetilde{x_{n}}+\widetilde{h_{n}}$ converges to $x+h$ and $\liminf c\left(\widetilde{\sigma_{n}}\right) \leq \psi_{0}(x)$. We then obtain that $P^{*}(x+h) \leq$ $\psi_{0}(x)$. We also know that $P^{*}(x+h) \geq \psi_{0}(x+h)=P^{*}(x)+\psi_{0}(h)$. Hence we have that $h=0$ and $x \in \overline{\operatorname{Im}(R)}$.

Proof of Theorem 4.2: If there exists $\psi_{0} \in K$ such that $P^{*}(x)=\psi_{0}(x)$ then by Theorem 4.1, $x \in \overline{\operatorname{Im}(R)}$. By definition of $P^{*}$ it is easy to show that, for all 
$\sigma \in \Sigma, P^{*}(R(\sigma)) \leq c(\sigma)$. Using the fact that $c$ is a linear form and that $P^{*} \circ R$ is sublinear, we obtain $P^{*} \circ R=c$ and the restriction of $P^{*}$ to $\operatorname{Im}(R)$ is a continuous linear form. Consequently, for all $y \in \operatorname{Im}(R)$ we have $P^{*}(y)=-P^{*}(-y)$ and this last result is also true for $x \in \overline{\operatorname{Im}(R)}$. Since $P^{*}(x) \geq \sup _{\psi \in K^{*}} \psi(x)$ and $-P^{*}(-x) \leq \inf _{\psi \in K^{*}} \sup _{\psi \in K^{*}} \psi(x)$, it is clear that $\{\psi(x): \psi \in K\}$ is reduced to a unique element. The converse is immediate. 


\section{References}

[1] Ansel, J-P. and C. Stricker (1994), "Couverture des actifs contingents et prix maximum," Annales de l'Institut Henri Poincaré, Probabilités et Statistiques, 30 (2), p. 303-315.

[2] Back, K., and S. Pliska (1990), "On the fundamental theorem of asset pricing with an infinite state space," Journal of Mathematical Economics, 20, p. 1-33.

[3] Bensaid, B., Lesne, J.P., Pagès, H., and J. Scheinkman (1991), "Derivative asset pricing with transaction costs," Mathematical Finance, 2, p. 63-86.

[4] Black, F., and M. Scholes (1973), "The pricing of options and corporate liabilities," Journal of Political Economy, 81, p. 637-54.

[5] Chen, Z. (1992), "Arbitrage pricing and optimal security design with transaction costs and taxes," University of Wisconsin mimeo.

[6] Constantinides, G. (1986), "Capital market equilibrium with transaction costs," Journal of Political Economy, 94, p. 842-62.

[7] Constantinides, G. (1993), "Option pricing bounds with transaction costs," University of Chicago mimeo.

[8] Cox, J., and S. Ross (1976), "The valuation of options for alternative stochastic processes," Journal of Financial Economics, 3, p. 145-66.

[9] Cvitanic, J., and I. Karatzas (1993), "Hedging contingent claims with constrained portfolios," Annals of Applied Probability, 3, p. 652-81.

[10] Davis, M., and A. Norman (1990), "Portfolio selection with transaction costs," Mathematics of Operations Research, 15, p. 676-713.

[11] Davis, M., Panas, V., and T. Zariphopoulou (1993), "European option pricing with transaction costs," SIAM Journal of Control and Optimization, 31, p. 470-93.

[12] Debreu, G. (1959), Theory of Value, Wiley: New York.

[13] Delbaen, F. and W. Schachermayer (1994a), "A general version of the fundamental theorem of asset pricing," Math. Annalen, 300, 463-520.

[14] Delbaen, F. and W. Schachermayer (1994b), "Arbitrage and free lunch with bounded risks for unbounded continuous processes," Mathematical Finance, $4,343-48$. 
[15] Duffie, D., and C.-F. Huang (1986), "Multiperiod security market with differential information," Journal of Mathematical Economics, 15, p. 283-303.

[16] Dybvig, P., and C.-F. Huang (1988), "Nonnegative wealth, absence of arbitrage, and feasible consumption plans," Review of Financial Studies, 1, p. 377-401.

[17] Dybvig, P., and S. Ross (1986), "Tax clienteles and asset pricing," Journal of Finance, 41, p. 751-62.

[18] Harrison, J., and D. Kreps (1979), "Martingales and arbitrage in multiperiod securities markets," Journal of Economic Theory, 20, p. 381-408.

[19] Harrison, J., and S. Pliska (1979), "Martingales and stochastic integrals in the theory of continuous trading," Stochastic Processes and their Applications, 11 , p. $215-60$.

[20] Harrison, J., and S. Pliska (1983), "A stochastic calculus model of continuous trading: complete markets," Stochastic Processes and their Applications, 15, p. $313-16$.

[21] He, H., and N. Pearson (1991), "Consumption and portfolio policies with incomplete markets and short-sale constraints: the infinite dimensional case," Journal of Economic Theory, 54, p. 259-304.

[22] Hindy, A. (1991), "Viable prices in financial markets with solvency constraints," Journal of Mathematical Economics, 24, p. 105-36.

[23] Jacka, S. (1992), "A martingale representation result and an application to incomplete financial markets," Mathematical Finance, 2, p. 239-50.

[24] Jameson, G. (1974), Topology and Normed Spaces, Chapman and Hall: London.

[25] Jouini, E., and H. Kallal (1995 a), "Martingales and arbitrage in securities markets with transaction costs," Journal of Economic Theory, 57(1), p. 17696.

[26] Jouini, E., and H. Kallal (1995 b), "Arbitrage in securities markets with short sales constraints," Mathematical Finance, 5(3), p. 197-232.

[27] Kreps, D. (1981), "Arbitrage and equilibrium in economies with infinitely many commodities," Journal of Mathematical Economics, 8, p. 15-35. 
[28] Leland, H. (1985), "Option pricing and replication with transaction costs," Journal of Finance, 40, p. 1283-1301.

[29] Prisman, E. (1986), "Valuation of risky assets in arbitrage- free economies with frictions," Journal of Finance, 41, p. 545-60.

[30] Ross, S. (1987), "Arbitrage and martingales with taxation," Journal of Political Economy, 95, p. 371-93.

[31] Schachermayer, W. (1992), "A Hilbert space proof of the fundamental theorem of asset pricing in finite discrete time", Insurance: Mathematics and Economics, 11, p. 249-257.

[32] Soner, H., Shreve, S., and J. Cvitanic (1995), "There is no nontrivial hedging portfolio for option pricing with transaction costs," Annals of Applied Probability, 5, p. 327-55.

[33] Stricker, C. (1990), "Arbitrage et lois de martingale," Annales de l'Institut Henri Poincaré, 26(3), p. 451-60. 